\title{
ANALYSIS OF TEMPORAL VARIATIONS IN TURBIDITY FOR A COASTAL AREA USING THE HILBERT-HUANG-TRANSFORM
}

\author{
Shigeru Kato ${ }^{1}$, Magnus Larson ${ }^{2}$, Takumi $\mathrm{Okabe}^{3}$ and Shin-ichi Aoki ${ }^{4}$
}

\begin{abstract}
Turbidity data obtained by field observations off the Tenryu River mouth were analyzed using the Hilbert-Huang Transform (HHT) in order to investigate the characteristic variations in time and in the frequency domain. The Empirical Mode Decomposition (EMD) decomposed the original data into only eight intrinsic mode functions (IMFs) and a residue in the first step of the HHT. In the second step, the Hilbert transform was applied to the IMFs to calculate the Hilbert spectrum, which is the time-frequency distribution of the instantaneous frequency and energy. The changes in instantaneous frequencies showed correspondence to high turbidity events in the Hilbert spectrum. The investigation of instantaneous frequency variations can be used to understand transitions in the state of the turbidity. The comparison between the Fourier spectrum and the Hilbert spectrum integrated in time showed that the Hilbert spectrum makes it possible to detect and quantify the cycle of locally repeated events.
\end{abstract}

Keywords: Hilbert-Huang Transform, instantaneous frequency, turbidity, Hilbert spectrum

\section{INTRODUCTION}

Field observations typically include many phenomena in time and space operating at a variety of scales. Some observations contain intermittency that has the characteristics of spike noise and a lack of measurements. Moreover, the effects nonlinear phenomena are frequently embedded in the data. Turbidity is typically a measurement parameter that shows high intermittency in observed time series. Therefore, it is difficult to analyze the characteristics of the temporal variation in turbidity using traditional methods such as Fourier transform (FT) analysis. Superimposed periodic functions (sine and cosine waves) are employed to represent the data in FT analysis. The application of the FT inherently imposes the limitations of linearity and stationarity on the analyzed data. Also, the FT provides information in terms of frequency, wheras the temporal information is lost. This property makes it impossible to investigate a phenomenon on a time axis. Recently, the short time Fourier transform (STFT) and the Wavelet transform (WT) have been applied as more effective methods for the analysis in the time-frequency domain. The analysis using STFT and the WT, however, has limitations on the resolution in frequency and time due to the Uncertainty Principle. In addition, these methods are still based on the Fourier analysis. It is often difficult to apply these methods to nonlinear and nonstationary data, similar to what is the case for the FT.

This study aims at investigating the characteristics of the temporal variation in turbidity using the Hilbert-Huang Transform (HHT) analysis. The turbidity data was obtained from field observations in a coastal area in southern Japan. The HHT was initially proposed by Huang et al. (1998) for nonlinear and non-stationary data analysis. In recent years, the HHT has been applied in many research fields, for example, mechanical engineering (Chen et al., 2007), earthquake engineering (Dong et al., 2008), structural engineering (Quek et al., 2005), fluid dynamics (Ding et al., 2007), molecular dynamics (Phillips et al., 2003), and financial analysis (Huang et al., 2003), as a useful method for the analysis of non-stationary and/or nonlinear data. In coastal engineering, a few applications have been reported (e.g., Veltcheva, 2002; Schlurmann et al., 2002; Datig and Schlurmann, 2004; Paul et al., 2005; Chen et al., 2009), but the focus has been on wave analysis. Analysis of turbidity data inherently contains many difficulties because there is high intermittency, often with much similarity to spiky noises, and HHT may be a suitable method to apply.

Thus, in the present study the HHT is applied to analyze turbidity data from a coastal area. The HHT enabled us to investigate the characteristics of temporal variations in turbidity in the timefrequency domain. Furthermore, the performance and effectiveness of the HHT analysis were demonstrated by comparison with the FT.

${ }^{1}$ Architecture and Civil Engineering, Toyohashi University of Technology, 1-1 Hibarigaoka, Tempaku-cho, Toyohashi, Aichi, 441-8580, Japan

${ }^{2}$ Water Resources Engineering, Lund University, Box 118, 22100 Lund, Sweden

${ }^{3}$ Architecture and Civil Engineering, Toyohashi University of Technology, 1-1 Hibarigaoka, Tempaku-cho, Toyohashi, Aichi, 441-8580, Japan

${ }^{4}$ Architecture and Civil Engineering, Toyohashi University of Technology, 1-1 Hibarigaoka, Tempaku-cho, Toyohashi, Aichi, 441-8580, Japan 


\section{FIELD OBSERVATIONS}

Field observations of waves, currents, and turbidity were conducted $2 \mathrm{~km}$ off the Tenryu River mouth in September, 2006 (Okabe et al., 2008). The Tenryu River is located in the center of the EnshuNada coast, which is one of the Pacific coasts of the main island of Japan (Figure 1). This coast is exposed to stormy wave conditions caused by typhoons in the summer and fall every year. Also, the reduction of the sediment supply from the Tenryu River has caused coastal erosion problems along this coast. There is a wrecked ship about $2 \mathrm{~km}$ off the Tenryu River mouth in a water depth of around about $20 \mathrm{~m}$. In order to measure the turbidity, an optical sensor was installed on the deck of the ship where the water depth is about $18 \mathrm{~m}$ below the mean sea surface. The turbidity was measured every 10 minutes using 30 samples at 1 second intervals as a proxy measure of suspended sediment concentration. Furthermore, sea surface elevations were recorded every 0.5 seconds using an ultrasonic-type wave gauge, and vertical profiles of 1-minute averaged current velocity at every 10 minutes were measured with an acoustic Doppler profiler during the observation period.

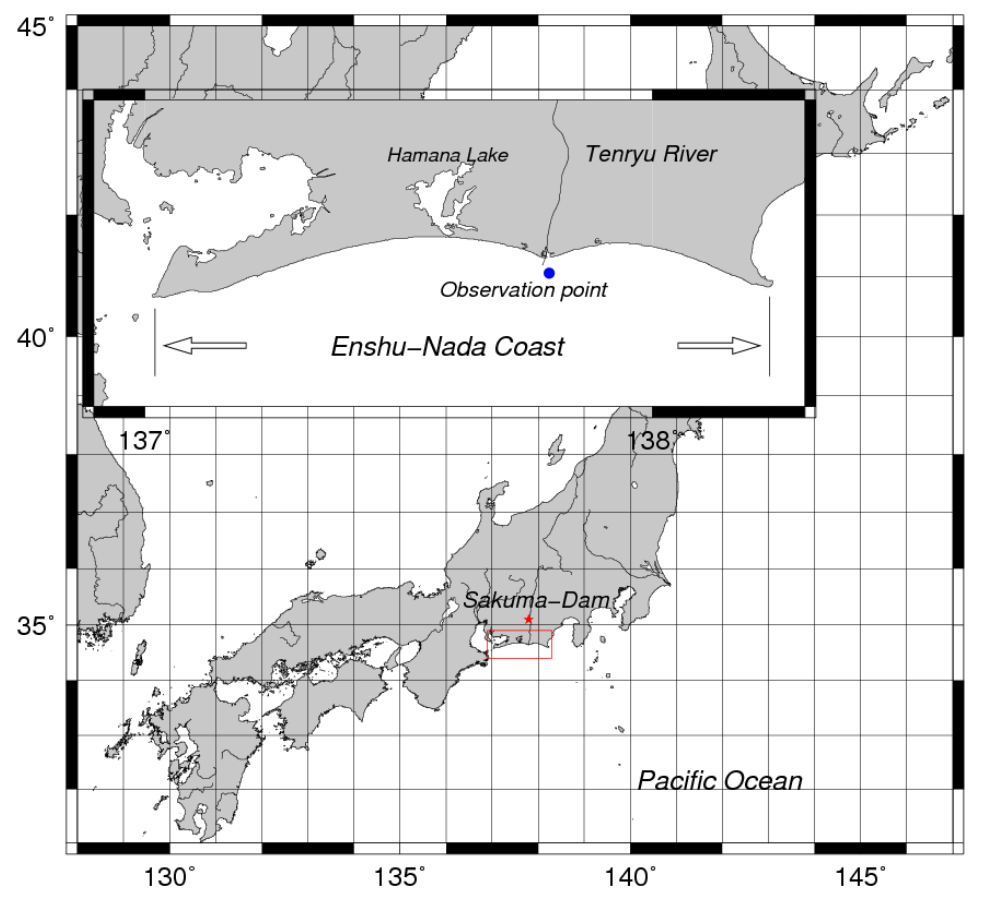

Figure 1. Field site (Enshu-Nada Coast) and observation point (2km off the Tenryu River mouth)

\section{SUMMARY OF HILBERT-HUANG TRANSFORM (HHT)}

The HHT mainly consists of two parts: empirical mode decomposition (EMD) and Hilbert transform (HT) (Huang et al., 1998). First, the original data are decomposed into a set of intrinsic mode functions (IMF), which satisfy the conditions of linearity and stationarity with distinct time scale and a residue. The residue represents a trend or average of the original data. Second, the HT is applied to each IMF to provide instantaneous properties, such as a frequency, amplitude, and energy, of the IMFs. Based on these variables, the Hilbert energy spectrum (HS) is obtained. The HS can illustrate the energy distribution and its variation in a time-frequency domain.

\section{Empirical Mode Decomposition (EMD)}

The original data are decomposed empirically into a finite number of IMFs and their residue using the assumption that any data consist of individual characteristic oscillations. The IMFs obtained by the empirical decomposition have to satisfy the following conditions for being a simple and locally symmetric oscillation mode:

- In the whole dataset, the number of extremes and the number of zero-crossings must either equal or differ at most by one.

- At any point, the mean value of the upper envelope, defined by the local maxima, and the lower envelope, defined by the local minima, is zero. 
Based on these definitions, it is possible that the IMFs have amplitude and frequency modulation. The IMFs are subject to Hilbert transform in the next step. The details of the EMD procedure to obtain the IMFs and residue from the original data will be referred to Huang et al. (1998) and others. The stopping criterion for the sifting process in EMD proposed by Rilling et al. (2003) was used in this study.

\section{Hilbert Transform (HT)}

The HT is a phase shift filter defined by Eq. (1). Through the HT, a sine function is converted into a cosine function. The analytic signal is defined by Eq. (2) using the original data series, $x(t)$, and the Hilbert transformed data series, $y(t)$.

$$
\begin{aligned}
& y(t)=p \cdot v \cdot \int_{-\infty}^{\infty} \frac{x(t-\tau)}{\pi} d \tau \\
& z(t)=x(t)+i \cdot y(t)=a(t) \cdot e^{i \theta} \\
& a(t)=\sqrt{x(t)^{2}+y(t)^{2}} \\
& \theta(t)=\arctan \left(\frac{y(t)}{x(t)}\right) \\
& f(t)=\frac{1}{2 \pi} \frac{d \theta(t)}{d t}
\end{aligned}
$$

where p.v. indicates the Cauchy principal value (Boashash, 1992), $a(t)$ and $f(t)$ are referred to as an instantaneous amplitude (IA) and an instantaneous frequency (IF) (Claerbout , 1976; Boashash, 1992 ), respectively.

In the HHT, the HT is applied to the IMFs and instantaneous variables such as IA and IF are obtained. Using these variables, the original data, $x(t)$, can be represented by, which is a general form of the Fourier expansion:

$$
x(t)=\sum_{j=1}^{n}\left[a_{j}(t) \exp \left(i \cdot 2 \pi \int_{0}^{T} f_{j}(t) d t\right)\right]+r_{n}(t)
$$

\section{Hilbert Spectrum (HS)}

Both the IA and the IF are functions of time. Thus, the IA can be written as a function of time and frequency, $a(t, f(t))$. This amplitude function is known as the Hilbert amplitude spectrum, and the squared amplitude is called the Hilbert energy spectrum. In this study, the energy spectrum is denoted the Hilbert spectrum (HS), $H(t, f)$. Based on HS, the marginal HS, $h(f)$, is defined as:

$$
h(f)=\int_{0}^{T} H(f, t) d t
$$

The $H(t, f)$ implies a higher likelihood for the existence of a wave with a frequency at a certain time. Whereas the Fourier spectrum describes the existence of a wave component with a frequency over the entire time span of the data, the $h(f)$ provides a measure of the total amplitude or energy contribution for each frequency value.

\section{DATA ANALYSIS OF TURBIDITY BY HHT}

Turbidity data were obtained during 22 days from the observations off the Tenryu river mouth. Figure 2 shows the time series of turbidity, water level (depth), and significant wave height for the observation period. The turbidity data indicate a wide range of fluctuations with values that are less than $10 \mathrm{ppm}$ in calm conditions, e.g., from 0 to 200 hours, and that may reach $100 \mathrm{ppm}$ in stormy conditions, such as from 360 to 420 hours. Because of these characteristics, the turbidity data are not suitable for Fourier-based analysis. Thus, the application of HHT was considered. In this study, a time period of 120 hours including events of high waves and high turbidity, covering the period from 340 to 460 hours in Figure 2, was used.

Figure 3 shows eight IMF components $\left(c_{1} \sim c_{8}\right)$ and the residue $\left(r_{8}\right)$ extracted by the EMD from the turbidity data. Figure 3 (a) is the original data of turbidity, and (b) (i) are IMF components and (j) is the residue. The data used as the input to the EMD are referred to as the "original data", or simply "data", in this paper. The difference between the data and the reconstructed data, which are the sum of $c_{1} \sim c_{8}$ and $r_{8}$, is shown in Figure 4. The difference is small, which is evidence of the completeness of the EMD method implying that the set of the IMF and the residue satisfy Eq. (8) derived theoretically. 

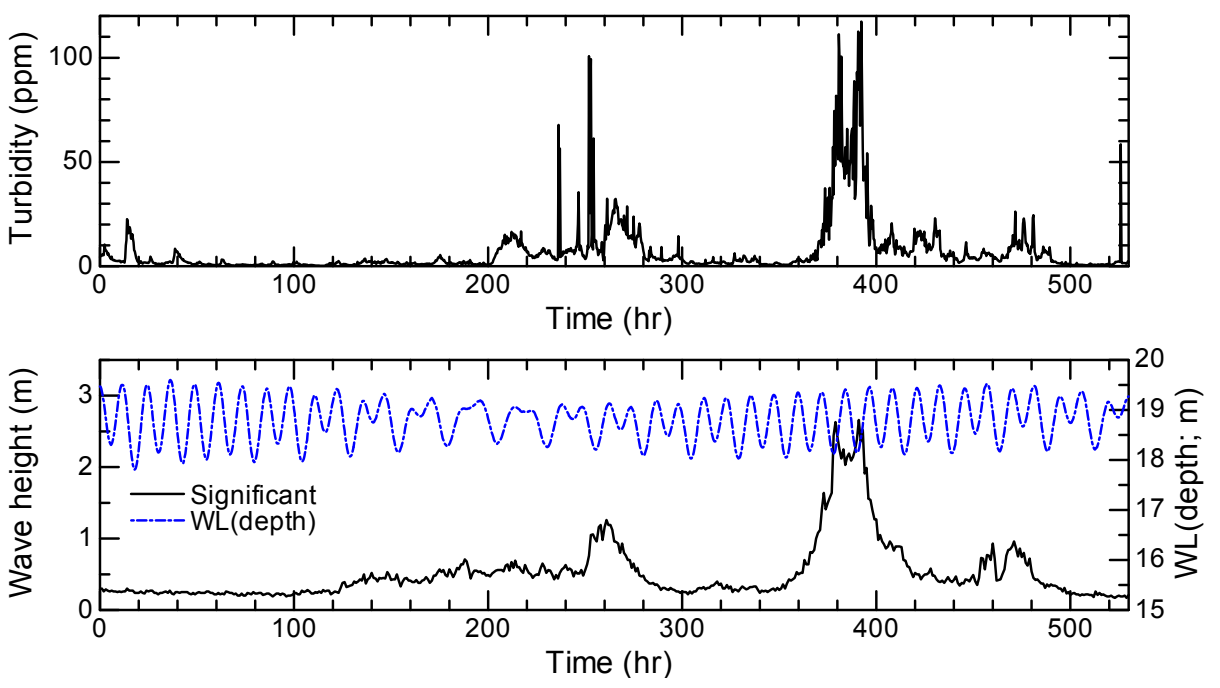

Figure 2. Time series of turbidity, water surface, and wave height during observations

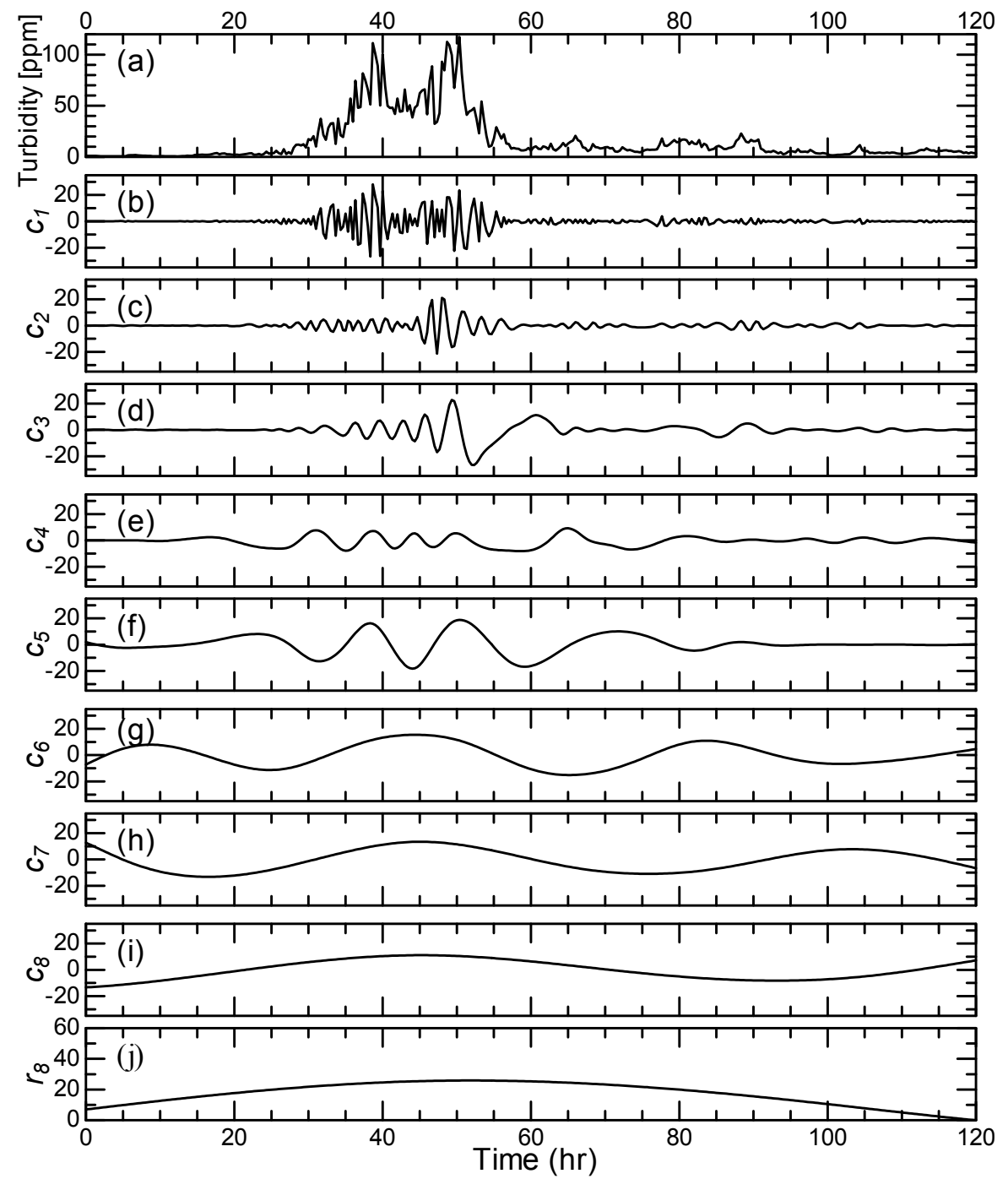

Figure 3. Turbidity data and Intrinsic Mode Functions (IMFs) extracted by Empirical Mode Decomposition (EMD); (a) original data on turbidity; (b) (i) IMFs of $1^{\text {st }} \sim 8^{\text {th }}$ mode $\left(c_{1} \sim c_{8}\right)$, and (j) residue $\left(r_{8}\right)$ 
Moreover, it is demonstrated that significant lack of information embedded in the data is not caused by the decomposition.

$$
\begin{aligned}
& \text { Reconstructed data : } x^{\prime}(t)=\sum_{i=1}^{n} c_{i}(t)+r_{n}(t) \\
& \text { Error (difference) : } \operatorname{Err}(t)=x(t)-x^{\prime}(t)
\end{aligned}
$$

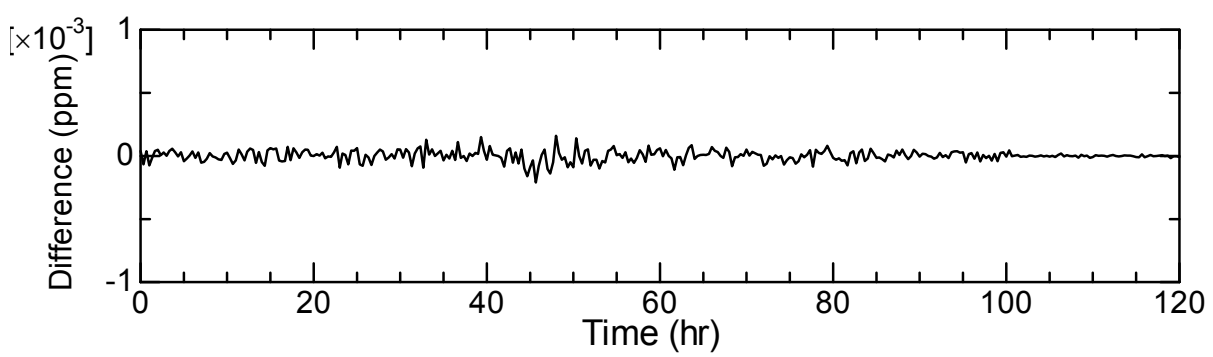

Figure 4. Difference (error) between the original data and reconstructed data based on the sum of all IMFs and the residue.

The time-frequency distribution of the HS is shown in Figure 5. The IFs change to high frequencies with increasing turbidity towards $T=40$ hours. In addition, after $T=50$ hours, the IFs change to low frequencies with decreasing turbidity. This tendency is confirmed not only in the high turbidity event at $T=40 \sim 50$ hours, but also in the small increase in turbidity at $T=65,80$, and 90 hours noted by investigating the IFs in details, though the magnitude of the energies at $T=65,80$, and 90 hours is much smaller than that at $T=40 \sim 50$ hours.
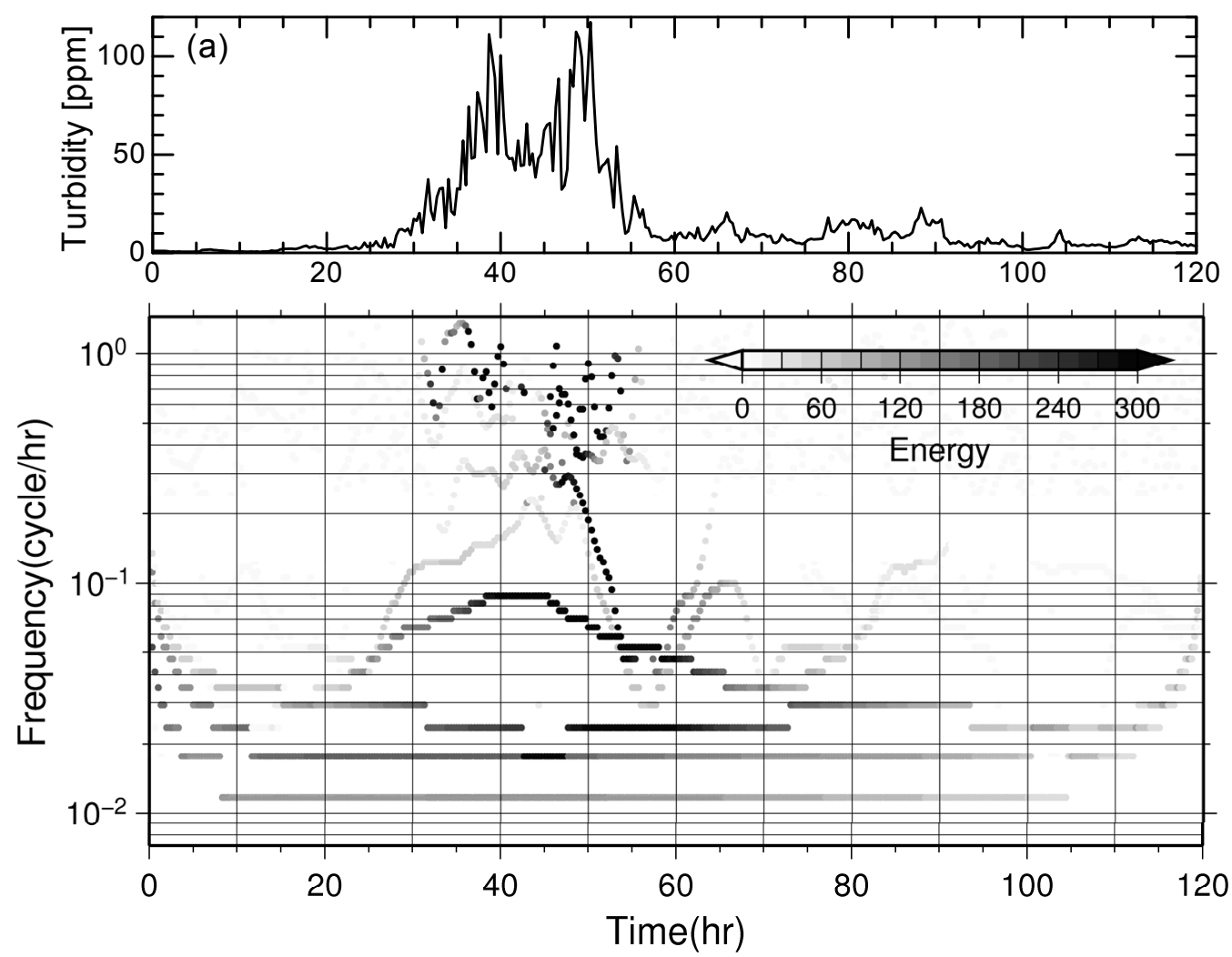

Figure 5. Time-frequency distribution for Hilbert spectrum of turbidity

Once turbidity becomes high, it does not decrease rapidly when the suspended matter is fine. Also, the variation in turbidity for decreasing values will be slower than that for increasing values. The 
increase in turbidity is generated directly by the reinforcement of external forces. Therefore, the variation in turbidity will be markedly affected by the fluctuations in wave and current conditions. It will induce the IF changes to high frequencies. On the other hand, the decrease in turbidity is mostly caused by diffusion and settling of suspended matter with slow and small fluctuations.

Figure 6 shows the marginal HS (MHS) calculated by Eq. (7) together with the Fourier Spectrum (FS). There is a peak at $f=0.088$ cycles/hour ( 11.3 hours $)$ in the MHS. This period corresponds to the interval between the high turbidity peaks at $T=38.7$ hours and 50.3 hours, which are easily identified in $c_{5}$. It is supposed that MHS can show the interval (or period) between high turbidity events that occur repeatedly and locally in time. However, in the FS, there are peaks before and after $f=0.088$ cycles/hour. This is the significant difference between the MHS and the FS.

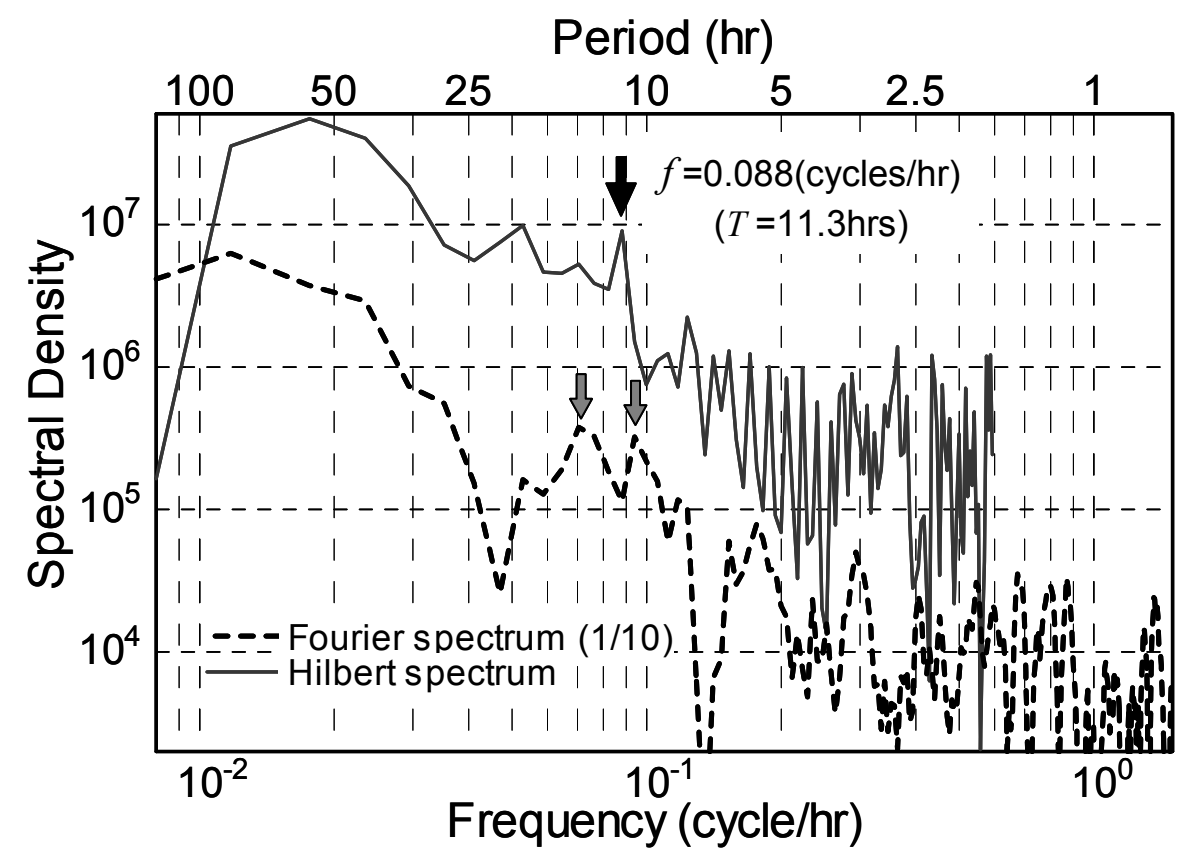

Figure 6. Marginal Hilbert spectrum and Fourier spectrum of turbidity; Fourier spectrum is multiplied by $1 / 10$ to shift the profile downward and to make a comparison easier.

\section{CONCLUSIONS}

- The empirical mode decomposition (EMD) could decompose the turbidity data obtained through field observation into a small number of IMFs and a residue, whereas the Fourier transform needs a large number of harmonic functions to represent the data.

- The instantaneous frequency showed the variation corresponding to the turbidity fluctuations in time. The change in frequency denotes one of the main characteristics of the local variation in time series data. Therefore, the investigation of the frequency variation can be undertaken to understand the properties of the turbidity fluctuations in detail.

- The marginal Hilbert spectrum could identify the cycle of an event, which is repeated locally, whereas the Fourier spectrum could not. This is the important difference between the Hilbert spectrum and the Fourier spectrum, and it is particularly useful when HHT is applied in nonstationary data analysis.

\section{ACKNOWLEDGEMENTS}

This study was supported by "Dynamic sediment management and coastal disaster prevention by advanced technologies", the Special Coordination Funds for Promoting Science and Technology of Ministry of Education, Culture, Sports, Science and Technology of Japan. The authors would like to thank members of Coastal Engineering Laboratory, Toyohashi University of Technology, Japan, for their support in field observations. 


\section{REFERENCES}

Boashash, B. 1992. Estimating and interpreting the instantaneous frequency of a signal - Part 1: Fundamentals, IEEE Proceedings, 80, 520-538.

Chen, H.G., Y.J.Yan and J.S. Jiang. 2007. Vibration-based damage detection in composite wingbox structures by HHT, Mechanical Systems and Signal Processing, Vol.21, 307-321.

Chen, Y. L., S. Y. Tzang and S. H. Ou. 2009. Preliminary application of the HHT to analyze the characteristics of nonlinear wave decay over a fluidized bed, Proceedings of Coastal Dynamics, CD-ROM (Paper No. 118).

Claerbout, J.F. 1976. "Transforms" in "Fundamentals of Geophysical Data Processing", Blackwell Scientific Publications, 1-23.

Datig, M. and T. Schlurmann. 2004. Performance and limitations of the Hilbert-Huang transformation (HHT) with an application to irregular water waves, Ocean Engineering, Vol.31, 1783-1834

Ding, H., Z. Huanga, Z. Songa and Y. Yanb. 2007. Hilbert-Huang transform based signal analysis for the characterization of gas-liquid two-phase flow, Flow Measurement and Instrumentation, Vol.18, $37-46$.

Huang, N.E., Z. Shen, S.R. Long, M.C. Wu, S.H. Shih, Q. Zheng, C.C. Tung and H.H. Liu. 1998. The empirical mode decomposition method and the Hilbert spectrum for non-stationary time series analysis. Proc. Roy. Soc. London, A454, 903-995.

Huang, N.E., M.L. Wu, W. Qu, S.R. Long and S.S.P. Shen. 2003. Applications of Hilbert-Huang transform to non-stationary financial time series analysis, Applied Stochastic Models in Business and Industry, Vol.19, 245-268.

Okabe, T., S. Aoki and S. Kato. 2008. Properties of long-period waves and velocity fluctuations near river mouth and narrow inlet, Proc. 31st Int. Conf. Coastal Eng., ASCE. (poster)

Paul, A.H., W.W. David and J.M. Kaihatu. 2005. A comparison of the energy flux computation of shoaling waves using Hilbert and Wavelet spectral analysis techniques, The Hilbert-Huang Transform in Engineering (Eds by N.E. Huang and N.O. Attoh-Okine), CRC Press, 83-96.

Phillips, S.C., R.J. Gledhill, J.W. Essex and C.M. Edge. 2003. Application of the Hilbert-Huang transform to the analysis of molecular dynamics simulations, Journal of physical chemistry, A, Vol.107, No.24, 4869-4876.

Quek, S.T., P.S. Tua and Q. Wang. 2005. Comparison of Hilbert-Huang, Wavelet, and Fourier Transforms for Selected Application, In "The Hilbert-Huang Transform in Engineering” (Edited by N. Huang and N. O. Attoh-Okine, published by CRC Press), 213-244.

Rilling, G., P. Flandrin and P. Goncalves. 2003. On empirical mode decomposition and its algorithms. In: IEEE-EURASIP workshop on nonlinear signal and image processing, Grado (I)

Schlurmann, T., M. Bleck and H. Oumeraci. 2002. Wave transformation at artificial reefs described by the Hilbert-Huang Transformation, Proceedings of the 28th International Conference on Coastal Engineering (ICCE 2002), 1791-1803.

Veltcheva, A.D. 2002. Wave and group transformation by a Hilbert spectrum, Coastal Engineering Journal, Vol.44, 283-300. 